Jean-Michel Jeannin

Beat Meier

\title{
Fortbildung
}

Schweiz Z Ganzheitsmed 2012;24:86-93

DOI: 10.1159/000337331

\section{Schweizerische Jahrestagung für Phytotherapie: Phytotherapie in der Neurologie}

Die 26. Schweizerische Jahrestagung für Phytotherapie behandelte ein Thema, das bisher kaum beachtet wurde: «Phytotherapie in der Neurologie». Diese Wahl forderte einigen Mut seitens der Organisatoren, der aber mit der Teilnahme von rund 250 Personen belohnt wurde. Auf der Tagung referierten praktisch tätige, erfahrene Neurologen und Pharmakologen. Dabei zeigte sich eine Vielzahl von Anwendungsmöglichkeiten der Phytotherapie, die namentlich bei der Behandlung von chronischen Zuständen einiges zu bieten hat.

unter dem Titel «Phytotherapie in der Neurologie - Bedürfnisse und Erfahrungen" mit einem Einblick in die Geschichte der Fachgebietes. Wichtige Fortschritte in der Neuroanatomie und in der klinischen Neurologie führten gegen Ende des 19. Jahrhunderts zur Verselbständigung der Disziplin und zur Gründung einer eigenen Fachgesellschaft. Die Erfindung der Computertomographie war ein für die weitere Entwicklung der Disziplin entscheidender Impuls.

Die Ätiologie und Pathologie vieler neurologischer Krankheitsbilder sind trotz intensiver Forschungen noch unklar. Dies erschwert die Entwicklung neuer Arzneimittel. Der klinische Neurologe ist derzeit stark auf schulmedizinische Methoden konzentriert, obwohl aus Untersuchungen bekannt ist, dass z.B. 27-100\% der Multiple-Sklerose-Patienten komplementäre Therapien einsetzen: Physikalische Therapie, Phytotherapie, Cannabis, Vitamine, Mineralien, Entspannungstechniken, Akupunktur und Massage wurden genannt. Die meist genannten Symptome, die mit solchen Methoden behandelt werden, sind Schmerzen, Müdigkeit und Stress. Das Interesse der Patienten an pflanzlichen Arzneimitteln und ihrer Anwendung nimmt $\mathrm{zu}$, sodass auch die Mediziner (Psychiater, Neurologen) sich mit deren Wirkungen, Nebenwirkungen und Kontraindika- tionen auseinandersetzen müssen. Die Anzahl der klinisch dokumentierten Anwendungen von Heilpflanzen bei Patienten mit neurologischen Erkrankungen ist derzeit noch sehr begrenzt.

Aus der eigenen Erfahrung des Referenten haben sich die Kombination von Baldrian und Hopfen sowie Zubereitungen mit Lavendelöl bei Insomnie, die nur in der Schweiz gängige Kombination Baldrian/Melisse/ Passionsblume/Pestwurz bei Angststörungen, Johanniskraut bei Depression und Ginkgo bei dementieller Entwicklung bewährt. Da sie gut verträglich sind, ist die Anwendung dieser pflanzlichen Arzneimittel bei geriatrischen Patienten, die oft mit einer grossen Anzahl von Medikamenten behandelt werden («Polypharmazie»), besonders sinnvoll.

«Cannabis-THC: Stützt neues Wissen die Erfahrung?»

Dr. med. Regula Spreyermann, leitende Ärztin, und Dr. med. Holger Lochmann, Oberarzt, beide vom Zentrum für Querschnittgelähmte und Hirnverletzte des Schweizerischen Paraplegikerzentrums an der REHAB Basel, präsentierten unter dem Titel «Cannabis-THC: Stützt neues Wissen die Erfahrung?» in einem zweiteiligen Referat Erfahrungen mit dem Einsatz von Cannabis-Wirkstoffen bei QuerSaller und Beat Meier für das wissenschaftliche Programm verantwortlich.

\section{KARGER}

Fax +497614520714 Information@Karger.de www.karger.com
(๑) 2012 S. Karger GmbH, Freiburg

Accessible online at: www.karger.com/szg
Prof. Dr. sc. nat. Beat Meie Geschäftsstelle SMGP

c/o Zürcher Hochschule für angewandte Wissenschaften Grüental, Postfach 335, 8820 Wädenswil, Schweiz

Tel. +41589345806

Beat.Meier@zhaw.ch 
schnittgelähmten zur Behandlung von Spastik und neuropathischen Schmerzen.

Regula Spreyermann startete mit den Verdiensten von Sir Ludwig Guttmann für das heute gültige Rehabilitationskonzept für Querschnittgelähmte. «Comprehensive Care» stellt die umfassende Betreuung für Menschen mit Querschnittlähmung ins Zentrum. Diese beinhaltet - nach einer stationären Erstrehabilitationsphase - die lebenslange Nachsorge.

Die wichtigsten medizinischen Probleme bei der Nachsorge sind Schmerzen, Spastik und rezidivierende Infektionen. $\mathrm{Zu}$ deren Behandlung stehen zwar Medikamente zur Verfügung. Deren Einsatz ist aber wegen ihrer Nebenwirkungen und/oder infolge nachlassender Wirksamkeit in der Dauertherapie limitiert. Aus internistischer Sicht ist es zudem nicht sinnvoll, Schmerzmittel lebenslang und hoch dosiert einzusetzen. Oft wollen die jungen Patientinnen und Patienten mit möglichst wenigen Medikamenten auskommen, damit sie im Alltag nicht beeinträchtigt sind. Die Erfahrung der Referentin zeigt, dass die Schulmedizin hier ihre Grenzen hat. Deshalb wird auch mit Phytotherapie und anderen erfahrungsmedizinischen Therapien wie Homöopathie, Neuraltherapie und Physiotherapie gearbeitet.

Im Rahmen der Nachsorge berichteten Patientinnen und Patienten über ein gutes Ansprechen der spastischen Symptome auf Cannabis. Da die Anwendung der herkömmlichen Antispastika wie Lioresal, Dantamacrin, Musaril und Valium sehr frustrierend ist, entstand in der Klinik die Idee, diesen Aussagen mittels einer wissenschaftlichen Studie nachzugehen. Die Verabreichung von Tetrahydrocannabinol (Marinol ${ }^{\circledR}$, mit Sonderbewilligung aus den USA eingeführt) in einer Dosis von mindestens 15-20 mg täglich zeigte einen statistisch signifikanten positiven Effekt auf die Spastik. Der Aussagewert dieser Studie ist einzig durch die geringe Anzahl auswertbarer Patientinnen und $\mathrm{Pa}$ tienten limitiert. Die verschärfte Gesetzgebung und die verstärkten polizeilichen Kontrollen in der Schweiz, insbesondere bezüglich des Eigenkonsums für Cannabis, führten dazu, dass das Thema Cannabis für den medizinischen Gebrauch trotz dieser Resultate unter den Tisch zu fallen drohte.

\section{«Einsatz von Cannabis-Wirkstoff im Ambulatorium bei Querschnitt- patientInnen heute - Spastik und neuropathische Schmerzen»}

Holger Lochmann widmete sich dem Thema "Einsatz von Cannabis-Wirkstoff im Ambulatorium bei QuerschnittpatientInnen heute - Spastik und neuropathische Schmerzen». Für fast alle an der REHAB Basel betreuten Patienten sind, unabhängig von der individuellen Diagnose, zwei Themen relevant: die Spastik und die neuropathischen Schmerzen. Die Spastik wird in erster Linie mit Physiotherapie und Ergotherapie sowie medikamentös behandelt. Alternativen sind Botox intramuskulär, die intrathekale Baclofenpumpe und neuroorthopädische Eingriffe. Für die Therapie neuropathischer Schmerzen werden Antiepileptika, oft in Kombination mit Opiaten, verwendet. Auch Schmerzmodulatoren aus der Reihe der Antidepressiva sind üblich. Die Toleranz der Patientinnen und Patienten gegenüber diesen klassischen Medikamenten ist besonders im fortgeschrittenen Alter vermindert. Dazu kommen ein Verlust der Wirksamkeit sowie Interaktionen mit neuen, zusätzlich verordneten anderen Medikamenten.

Als Add-on- bzw. als Last-LineTherapie wird am Paraplegikerzentrum das THC-Präparat Dronabinol ${ }^{\circledR}$ verordnet. Nach Beobachtungen des Referenten sprechen Querschnittgelähmte zu etwa $50 \%$ an. In optimalen Fällen werden sowohl die Spastik als auch die neuropathischen Schmerzen positiv beeinflusst, zentrale Neben- wirkungen fehlen. Die häufig anzutreffende Blasen-Detrusor-Überaktivität-eineerwünschteZusatzwirkung - wird vermindert. Bei kachektischen Patientinnen ist die Steigerung des Appetits eine weitere erwünschte Wirkung, nicht aber bei primär übergewichtigen Personen im Rollstuhl: Hier führt eine Zunahme des Gewichts zum Abbruch des Therapieversuchs. Lochmanns Bilanz: «Insgesamt steht mit Dronabinol eine interessante Möglichkeit zur Behandlung unserer Patientinnen und Patienten zur Verfügung, welche jedoch nur in ausgewählten Fällen zur Anwendung kommt und einen deutlich erhöhten administrativen Aufwand für den verschreibenden Arzt zur Folge hat.» Neben einer Revision der Gesetzgebung müssten die Kosten einer Dronabinol-Therapie dringend gesenkt werden. In der Diskussion wies Prof. Dr. Ruedi Brenneisen, Universität Bern, auf die neuen Regelungen hin, die voraussichtlich ab 1. Juli 2012 in der Schweiz in Kraft treten. Sie werden den Zugang zu den medizinisch begründeten Cannabistherapien vereinfachen.

«Mild Cognitive Impairment (MCI): nur eine Trenddiagnose?»

«Es darf nicht sein, dass Menschen mit einer Demenzkrankheit Angst davor haben, in einen würdelosen Zustand abzugleiten.» Mit diesem Aufruf liess Dr. med. Irene BoppKistler (Abb. 2) von der Memory Klinik, Klinik für Akutgeriatrie des Stadtspital Waid in Zürich ihre Zuhörer verschiedene Ereignisse der letzten Monate unter dem Titel «Mild Cognitive Impairment (MCI): nur eine Trenddiagnose?» reflektieren, so auch den Freitod eines Prominenten. Sie machte von vornherein klar, dass der Mediziner vor einem grossen Problem steht und hier nicht etwa, wie in einer renommierten Zeitung zu lesen war, eine neue Krankheit mit Potential für Karrieren, Medikamente und Diagnostika etabliert wird. Eine Demenz- 


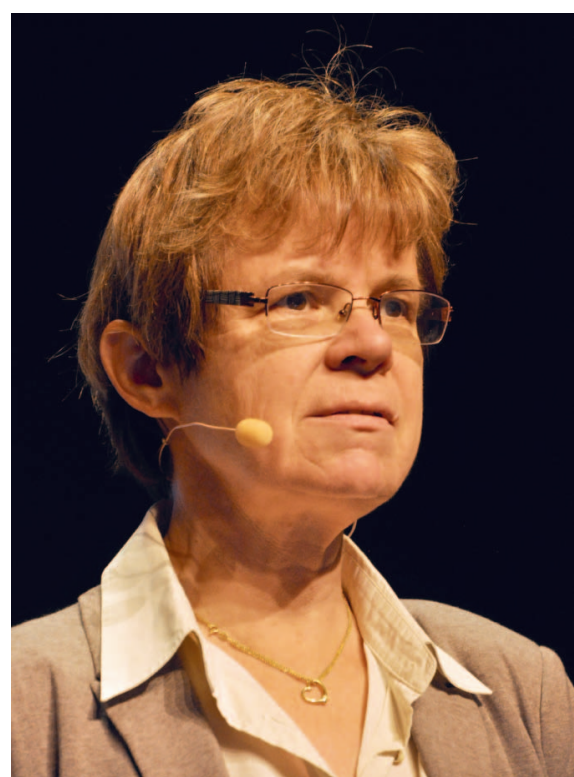

Abb. 2. Frau Dr. med. Irene Bopp-Kistler hielt ein flammendes Plädoyer für die Früherkennung und richtige Diagnosestellung bei dementiellen Erkrankungen zugunsten des Patienten, dem mit einer richtigen Betreuung und Aufklärung viel geholfen werden kann.

erkrankung beginnt oft unbemerkt mit einer subjektiven Gedächtnisstörung, die später in eine milde Hirnleistungsstörung (MCI) übergeht. Unter einem MCI wird eine milde kognitive Beeinträchtigung verstanden, die keine wesentliche Einschränkung im Alltag mit sich bringt. Ist das Gedächtnis gestört, spricht man von einem «amnestic MCI» (aMCI). Das MCI geht nicht bei allen Patienten in eine Demenz über. Der Verlauf ist oft stabil, auch eine Verbesserung oder gar eine Normalisierung sind möglich. Das aMCI ist aber oft die Vorstufe zu einer Alzheimer- oder einer anderen Demenzerkrankung. Zu Beginn letzterer stehen neuropsychologische Defizite im Vordergrund, bei der Lewy-BodyDemenz beispielsweise visuokonstruktive Störungen und Wahrnehmungsstörungen im Raum. Biomarker zum Nachweis der Krankheiten und zur Prognostik bezüglich des Verlaufs werden gegenwärtig intensiv erforscht. Bopps Erfahrung zeigt, dass es aber noch zu früh ist, sie in der Routinediagnostik einzusetzen.

Eine Demenz beginnt schleichend, im Anfangsstadium sind die Fehlleistungen sehr diskret. Daher kann die betroffene Person ihre Defizite nicht richtig einordnen. Steht sie noch im Berufsleben, fühlt sie sich möglicherweise als Mobbingopfer. Es kommt auch im Privatleben zu Kränkungen. Hier ist eine frühzeitige Abklärung gefordert. Eine klare Diagnose ist eine Erleichterung, auch wenn sie schwer wiegt. Sie verbessert das Verständnis für den betroffenen Patienten, Konflikte werden entschärft. Die Diagnose «MCI» kann somit nie nur eine Trenddiagnose sein. Eine frühzeitige Abklärung hilft, eine kognitive Störung richtig einzuordnen. Leichte kognitive Störungen können z.B. auch im Rahmen einer Depression auftreten. Da immer eine somatische Ursache hinter einem MCI stecken kann, müssen Klagen der Patienten über einen Verlust von Gedächtnisleistung stets ernst genommen werden. In einem gut abgestützten sozialen Umfeld und bei einer optimalen Unterstützung kann der Verlauf der Krankheit gemindert werden. Ärztinnen und Ärzte sind gefordert. Die richtige Diagnose steigert nach den Erfahrungen der Referentin das Verständnis für die Schwierigkeiten des Patienten im Alltag, womit Konflikte entschärft werden. Zudem können Patienten mit gezielten Massnahmen ihre CopingStrategien stärken und dank ihrer durchaus noch vorhandenen Lernfähigkeiten Kompensationsstrategien entwickeln.

Der Einsatz von Medikamenten ist beschränkt. Sowohl zum Einsatz von Acetylcholinesterase-Hemmern als auch zur Anwendung von Ginkgo biloba bei MCI-Patienten liegen gegenwärtig kontroverse Ergebnisse vor. Erstere werden infolge der Neben- wirkung von der Referentin nicht empfohlen. Da die Therapie mit Ginkgo kaum zu Nebenwirkungen führt, kann sie versucht werden. Sie hat namentlich in der Behandlung einer subjektiven Gedächtnisstörung ihren Wert. Im Nebensatz empfahl die Referentin Ginkgo auch zur Leistungssteigerung im Studium als sinnvolle Alternative zur problematischen, dennoch weit verbreiteten Anwendung von Ritalin.

«Vorteile pflanzlicher Sedativa eine Abschätzung anhand pharmakologischer und klinischer Profile» Prof. Dr. Jürgen Drewe, Universitätsspital Basel, hielt ein Referat mit dem folgenden Titel: «Vorteile pflanzlicher Sedativa - eine Abschätzung anhand pharmakologischer und klinischer Profile». Die Häufigkeiten von affektiven Störungen sowie die von Angstund Schlafstörungen nehmen $\mathrm{zu}$ und werden, auch weil die Anforderungen an das Individuum im Beruf und in der Gesellschaft steigen, gesellschaftlich immer wichtiger. Depressive Symptome und Schlafstörungen verstärken sich wechselseitig. 57\% der Patienten mit Angststörungen und Panikattacken und $54 \%$ der Patienten mit einer Depression vertrauen gemäss einer Studie pflanzlichen Arzneimitteln und komplementären Behandlungsmethoden, sowohl als alleinige Methode als auch in Kombination mit synthetischen Medikamenten. Nur wenige dieser pflanzlichen Präparate sind weltweit gesehen als Arzneimittel zugelassen, andere sind aufgrund eines gut dokumentierten langen Gebrauchs als traditionelle Heilmittel anerkannt, ohne dass die klinische Wirksamkeit durch gut kontrollierte, randomisierte, klinische Studien nachgewiesen wurde.

Das umfangreichste Datenmaterial existiert für Johanniskraut. 18 randomisierte, kontrollierte Studien sind veröffentlicht. Zwei Metaanalysen 
zeigten eine statistisch signifikante Überlegenheit gegenüber Placebo und eine therapeutische Wirksamkeit, die mit der von klassischen Antidepressiva (tri- und tetrazyklische Antidepressiva und SSRI) vergleichbar ist. Die Häufigkeit von klinisch relevanten unerwünschten Wirkungen war statistisch signifikant niedriger. Metaanalysen gibt es auch für die Anwendung von Baldrian, dessen Extrakte in Vergleichsstudien ebenso gut abschneiden wie Benzodiazepine, ohne deren Nebenwirkungspotential aufzuweisen. Die verbesserte Schlafqualität wird nicht durch die Probleme eines «Hang-Over» relativiert. Das Evidenzniveau ist trotzdem deutlich geringer als bei Johanniskraut, da die Zahl an gut geplanten, modernen Studien noch zu gering ist und viele kleinere Studien mit schlechter Bewertung und/oder schlechten Resultaten das Gesamtergebnis beeinflussen. Etabliert hat sich in den letzten Jahren die Kombination von Baldrian und Hopfen, bei der nach neusten pharmakologischen Forschungen sich ergänzende Wirkprinzipien (Baldrian aktiviert den Adenosin-A-Rezeptor und Hopfen bindet an den Melatonin-Rezeptor) zum pharmakologisch begründeten Erfolg führen. Der Referent schloss mit der Folgerung, dass «Phytopharmaka für einige neurologische Erkrankungen bei leichter bis mittelschwerer Symptomatik eine evidenzbasierte und gut verträgliche Alternative für klassische synthetische Medikamente darstellen».

\section{«Pflanzliche versus synthetische} Arzneimittel in der Geriatrie - eine Betrachtung zur Arzneimittelsicherheit aus pharmakologischer und pharmakokinetischer Sicht»

Prof. Dr. Dieter Loew, Wiesbaden, präsentierte unter dem Titel «Pflanzliche versus synthetische Arzneimittel in der Geriatrie - eine Betrachtung zur Arzneimittelsicherheit aus phar- makologischer und pharmakokinetischer Sicht» bedenkenswerte Fakten: Der Anteil der betagten Menschen an der Gesamtbevölkerung nimmt stetig zu. In Deutschland sind derzeit 8,2\% der Bevölkerung über 75 Jahre alt. Bis im Jahr 2050 rechnet man mit mehr als $10 \%$ an Patienten, die älter als 80 Jahre sind. Im Alter gibt es typische physiologische Veränderungen und typische multiple Krankheiten, die bei der medikamentösen Behandlung der Patienten und Patientinnen zu berücksichtigen sind. Zum Beispiel besteht eine Hypalbuminämie mit der Gefahr von Überdosierung, Wechselwirkungen und entsprechend auch Nebenwirkungen. Betagte werden häufig mit einer Vielzahl von Medikamenten behandelt. Von den häufig angewandten synthetischen Medikamenten wurden in der PRISCUS-Liste der potentiell inadäquaten Medikation für ältere Menschen 83 Arzneistoffe als potentiell inadäquat, 46 als fraglich und nur 26 als unbedenklich eingestuft. Die Vorteile von pflanzlichen Arzneimitteln, namentlich bei der Anwendung beim alten Patienten, sind vielfältig: Deren Proteinbindung ist im Allgemeinen gering, entsprechend besteht kaum Gefahr von Überdosierungen durch Verdrängung anderer Arzneimittel aus der Proteinbindung. Xenobiotika werden im Allgemeinen in der Leber durch CYP450 entgiftet. In Arzneipflanzen selten vorkommende problematische Bestandteile lassen sich mit entsprechenden Herstellungsverfahren eliminieren, so z.B. die Pyrrolizidinalkaloide. Mit der Ausnahme von vereinzelten Tubulusirritationen durch hochdosiertes Wacholderöl sind keine glomerulotoxischen oder tubulotoxischen Schädigungen, keine interstitiellen Reaktionen und keine Kumulation bei Niereninsuffizienz durch pflanzliche Arzneimittel bekannt: «Ich kenne keinen toxischen Metaboliten, der aus einer pflanzlichen Zubereitung entsteht», betonte Löw. Phytopharmaka sind Naturstoffe. Ihre Resorption, Biotransformation, Proteinbindung und Elimination verlaufen anders als bei synthetischen Arzneimitteln und deshalb problemloser. Sie bieten sich daher bei der Behandlung von Betagten als Alternative zu potentiell inadäquaten Synthetika an. In Tabelle 1 sind die Empfehlungen von Loew aufgeführt.

«Kopfschmerzen - welches Potential haben phytotherapeutische Behandlungen?»

In seinem Referat «Kopfschmerzen welches Potential haben phytotherapeutische Behandlungen?» wies Dr. med. Charly Gaul, vom Westdeutschen Kopfschmerzzentrum, Universitätsklinikum Essen, darauf hin, dass $81 \%$ der Patienten mit primären Kopfschmerzen (meist Migräne) Erfahrung mit komplementärmedizinischen Methoden haben, so auch mit Phytotherapie. In der Kopfschmerzbehandlung muss die Akuttherapie von Attacken von der vorbeugenden Behandlung mit dem Ziel der Reduktion der Attackenhäufigkeit differenziert werden. Mit den am besten wirksamen Substanzen erzielt man nur bei $60 \%$ der Patienten eine Schmerzminderung $2 \mathrm{~h}$ nach der Einnahme (Triptane) und nur bei 45\% eine Reduktion der Schmerzattacken um die Hälfte (mit Topiramat oder einem Betablocker). Deshalb befinden sich die Patienten ständig auf der Suche nach der «optimalen» Lösung. Die Phytotherapie bietet Möglichkeiten, von denen einige auch klinisch untersucht worden sind.

So wurde die Wirksamkeit von Pfefferminzöl in zwei randomisierten Studien untersucht: Die Wirkung einer lokalen Applikation einer 10\%igen Menthol-Lösung bei Kopfschmerzen vom Spannungstyp setzte bereits nach 15 min ein und steigerte sich über $60 \mathrm{~min}$. Sie war dem Placebo 
Tab. 1. Pflanzliche Arzneimittel als Alternative zu synthetischen Arzneimitteln beim alten Patienten

\begin{tabular}{lll}
\hline Indikation & Chemisch definierte Substanz & Pflanzliches Arzneimittel aus \\
\hline Chronische Herzinsuffizienz NYHA II & Diuretika, Betablocker, ACE-Hemmer, & Crataegus-Blätter mit Blüten \\
& AT $_{1}$-Antagonisten & \\
Unruhe, Schlafstörung & Hypnotika, Benzodiazepine & Baldrian, Kombinationen mit Baldrian (z.B. Hopfen) \\
Leichte und mittlere Depression & Tri-, Tetrazyklika, SSRI, SNRI, SSNRI, & Johanniskraut \\
& MAO-Hemmer & \\
Hirnleistungsstörung & Acetylcholinesterase-Hemmer, Memantin & Ginkgo biloba \\
Periphere arterielle Verschlusskrankheit & Buflomedil, Naftidrofuryl, Pentoxifyllin & Ginkgo-biloba-Extrakt \\
Reizmagen-Reizdarm-Syndrom & Metoclopramid, Domperidon, Alizaprid & Iberogast ${ }^{\circledR}$, Pfefferminzöl, Kombination \\
& & $\begin{array}{l}\text { Pfefferminzöl/Kümmelöl } \\
\text { Benignes Prostata-Syndrom }\end{array}$ \\
& $\alpha$-Rezeptorenblocker, $\alpha$-Reduktasehemmer & Sägepalme, Brennnesselwurzel, Roggenpollen, \\
& & Kürbissamen \\
Klimakterische Beschwerden & Östrogene, Gestagene & Cimicifuga racemosa, sibirischer Rhabarber \\
Spannungskopfschmerz & ASS, Paracetamol & lokal 10\%iges Pfefferminzöl \\
Arthrosen, Wirbelkörper-Syndrom, Myalgie & Nichtsteroidale Antiphlogistika, Analgetika, & Teufelskralle, Weidenrinde, Capsicum \\
& Myotonolytika & \\
\hline
\end{tabular}

überlegen und gleich wirksam wie perorales Paracetamol. Die Lösungen wurden jeweils zweimal auf die Stirn und auf die Schläfen aufgetragen. Die Wirksamkeit bei Migräne wurde gegen eine 0,5\%ige MentholLösung als Placebo geprüft. Nach $2 \mathrm{~h}$ waren $38,3 \%$ der mit dem Verum behandelten Patienten schmerzfrei, gegenüber $12,1 \%$ der mit dem Placebo behandelten Patienten. In einer anderen Untersuchung war eine Kombination von Pfefferminzöl und Eukalyptusöl besser wirksam als die Einzelsubstanzen allein.

Die Pestwurz ist unter den zur Migräneprophylaxe verwendeten Phytopharmaka am besten untersucht. Die Wirkung beruht auf einer Hemmung der COX-2- und in geringem Mass der COX-1-Synthese. Zudem wirkt Pestwurz spasmolytisch. Pestwurz wird in den Deutsch-Österreichischen-Schweizerischen und in den Europäischen Leitlinien zur Migränetherapie gegenwärtig als Prophylaktikum zweiter Wahl angeführt. Ein mit dieser Indikation zugelassenes Präparat ist allerdings nicht verfügbar.

Für das Mutterkraut (Tanacetum parthenium) liegen Studien mit einem $\mathrm{CO}_{2}$-Extrakt zur Prophylaxe von Migräneattacken vor. Möglicherweise sind die darin enthaltenen Parthenolide für die Wirksamkeit verantwortlich. In einer Studie mit einer optimalen Dosierung des Extrakts wurde die Anzahl Migräneattacken statistisch signifikant von 4,8 auf 2,9 pro Monat verringert: Die Reduktion der Attackenhäufigkeit war bei 30,3\% der mit dem Verum behandelten Patienten und bei $17,3 \%$ der mit dem Placebo behandelten Patienten grösser als 50\%. Gemäss den Richtlinien der International Headache Society gelten diese $\mathrm{Pa}$ tienten somit als Responder. Auch dieser Extrakt steht in der Schweiz nicht in Form eines zugelassenen Arzneimittels zur Verfügung.

Sporadisch berichten Patienten über Wirksamkeit von Cannabis zur Behandlung von Clusterkopfschmerzen-Attacken. Studien zum Einsatz der Hanfpflanze bei der Indikation «Clusterattacken» fehlen aber.

In der Praxis steht damit eigentlich nur das Pfefferminzöl uneingeschränkt zur Verfügung, sowohl zur Behandlung des Kopfschmerzes vom Spannungstyp als auch zur Behandlung der Migräne.

\section{«Lokale Behandlung der}

Polyneuropathie mit Capsaicin»

Dr. med. Stefan Hägele-Link begann seinen Vortrag über die "Lokale Behandlung der Polyneuropathie mit Capsaicin» mit der Beschreibung des Krankheitsbildes der Polyneuropathie. Diese ist eine generalisierte
Erkrankung des peripheren Nervensystems, welches von motorischen, sensiblen und autonomen Nerven mit ihren Schwann-Zellen, Ganglienzellen, ihrem Peri- und Epineurium sowie den dazugehörenden Blut- und Lymphgefässen gebildet wird. Die typischen Symptome der Polyneuropathie sind sensible Reizund Ausfallserscheinungen, motorische Schwäche, Muskelzuckungen, Krämpfe und Atrophien. Die häufigste Ursache für eine Polyneuropathie ist der Diabetes mellitus. In über $20 \%$ der Fälle kann die Ursache trotz ausführlicher Abklärung nicht ausfindig gemacht werden. Gelingt es nicht, die kausale Ursache $\mathrm{zu}$ identifizieren, steht die symptomatische Therapie im Vordergrund, namentlich die Bekämpfung des Schmerzes. Dazu stehen Antikonvulsiva mit Wirkung auf neuronale Calcium-Kanäle wie Gabapentin und Lamotrigin zur Verfügung. Auch Antidepressiva wie Amitryptilin und selektive Serotonin- und Noradrenalin-Wiederaufnahmehemmer sowie lang wirksame Opioide werden eingesetzt. Die topische Behandlung neuropathischer Schmerzen mit Capsaicin ist besonders vielversprechend. Capsaicin ist ein Alkaloid aus der Paprikapflanze. Am weitesten verbreitet ist Capsicum annuum. Capsicum ist eine krautige Blütenpflanze, die in glühender Sonne ge- 
deiht und sehr kälteempfindlich ist. Die Früchte sind langgedehnt, deren Farben reichen von Gelb bis Rot und über Violett bis fast Schwarz mit grosser Variationsbreite, wobei grüne Früchte immer unreif sind. Die pharmakologische Wirkung von Capsaicin beruht auf einer Aktivierung des sogenannten TRPV1(Transient Receptor Potential Vanilloid-1)Rezeptors, der einen nichtselektiven Kationenkanal darstellt. Der Rezeptortyp spielt im zentralen und im peripheren Nervensystem eine zentrale Rolle bei der Verarbeitung von sensorischen Prozessen. Derselbe Kanal wird ausser von Capsaicin auch durch Temperaturen über $42{ }^{\circ} \mathrm{C}$ stimuliert. Die langfristige Zuführung einer niedrigen Dosis oder eine kurzfristige Zuführung einer hohen Dosis von Capsaicin führt zu einem histologisch nachweisbaren «Rückzug» der für die Schmerzwahrnehmung verantwortlichen Nozizeptoren. Damit wird die protrahierte Dauer der Schmerzlinderung erklärt. Capsaicin wird typischerweise als Pflaster oder als Creme appliziert und ist in unterschiedlicher Dosierung für verschiedene Formen der Schmerztherapie zugelassen. Die einmalige, topische Applikation einer 8\%igen Zubereitung führt nach neusten Erkenntnissen $\mathrm{zu}$ einer 3 Monate anhaltenden Schmerzlinderung. Das Präparat ist für die Anwendung bei der peripheren Neu- ropathie mit Ausnahme der diabetischen Neuropathie vorgesehen. In den Leitlinien zur Therapie der schmerzhaften diabetischen Neuropathie wird Capsaicin in Konzentrationen von $0,075 \%$ im Sinne einer Level-B-Evidenz empfohlen. Auch aufgrund eigener Erfahrungen zog der Referent folgende Bilanz: «Zusammenfassend stellt Capsaicin eine interessante und erfolgversprechende Ergänzung der uns zur Verfügung stehenden therapeutischen Möglichkeiten zur Behandlung neuropathischer Schmerzsyndrome dar, welche in Zukunft möglicherweise an Bedeutung gewinnen wird.» Während die Applikation der traditionellen Formulierungen des stark brennenden und die Schleimhäute reizenden Capsaicins (auch in Extrakten) keine grossen Probleme verursacht, erfordern höhere Konzentrationen entsprechende Vorsichtsmassnahmen. Insbesondere die Augen können sehr empfindlich auf Capsaicin in Form einer «Schneeblindheit» reagieren.

\section{"L-Dopa in Arzneipflanzen und deren} Anwendung für Parkinsonpatienten» Den Stand des Wissens bezüglich «L-Dopa in Arzneipflanzen und deren Anwendung für Parkinsonpatienten» resümierte Dr. rer. nat. Klaus Peter Latté, Landeslabor Berlin-Brandenburg, Berlin. Der Morbus Parkinson ist die zweithäufigste neurodege- nerative Erkrankung. Weltweit sind etwa $1 \%$ der über 60 -Jährigen und $3 \%$ der über 80 -Jährigen betroffen. Im Verlauf der Krankheit nimmt die Dopamin-Konzentration in bestimmten Hirnarealen, in der Substantia nigra und im Striatum ab. Zur Behandlung stehen zurzeit folgende Möglichkeiten zur Verfügung:

- L-Dopa: Da Dopamin die BlutHirn-Schranke nicht passiert, kann es nicht zur Therapie des M. Parkinson verwendet werden. Das linksdrehende Levodopa (LDopa) vermag hingegen ins Hirn zu gelangen, wo es durch Decarboxylierung in Dopamin umgewandelt wird. Es muss aber zwecks Vermeidung von peripheren Nebenwirkungen mit peripheren Decarboxylase-Hemmern kombiniert werden.

- Dopamin-Agonisten (z.B. Lisurid, Ropinirol, Pramipexol);

- Monooxidase-B-Inhibitoren (Selegilin, Rasagilin);

- Inhibitoren der Dopamin-Antagonisten (Biperiden, Procyclidin) oder N-Methyl-D-Aspartat-Rezeptor-Antagonisten (Amantadin, Budipin).

Die lang dauernde Einnahme von L-Dopa führt bei nahezu allen Patienten $\mathrm{zu}$ einem Wirkungsverlust und zu motorischen Störungen, weshalb besser verträgliche Formen von L-Dopa gesucht werden. Einer der Ansätze befasst sich mit L-Dopa-hal-

\section{Fort- und Weiterbildung in Phytotherapie}

Hauptaufgabe der SMGP ist die Gewährleistung und Sicherstellung einer wissenschaftlich orientierten Ausbildung. Haben Sie Lust, an einem spannenden und abwechslungsreichen Angebot während 3 Jahren mitzumachen? Mit Ihnen noch unbekannten Kolleginnen und Kollegen in dieser Zeit Fäden zu spinnen? Die Phytotherapie als Mittler zwischen Schul- und Komplementärmedizin bietet sich geradezu an, neue Wege zu beschreiten. Wir garantieren für Kurse auf hohem Niveau, spannende Diskussionen und Auseinandersetzungen. Der Bezug zur Natur wird dabei nicht vergessen: Die Pflanzen werden auf Exkursionen an ihrem natürlichen Standort betrachtet. Die Ausbildung endet mit dem Fähigkeitsausweis Phytotherapie (SMGP), der von der FMH als auch von der FPH anerkannt ist. Details zur Weiterbildung mit den Terminen und die entsprechenden Reglemente finden Sie jederzeit aktuell auf www.smgp.ch. Der Zyklus ist modular aufgebaut, ein Einstieg deshalb jederzeit möglich. Ideal für den Start ist der Grundkurs, der jeweils von Donnerstag bis Samstag in Engelberg stattfindet. 2012 wird der Kurs vom 7. bis 9. Juni angeboten. 
tigen Pflanzen. Die Substanz wurde schon vor 100 Jahren aus Vicia faba (Ackerbohne) isoliert.

$V$. faba (Fabaceae) enthält in den Samen und Hülsen 0,25-0,5\% L-Dopa, daneben essentielle Aminosäuren und Proteine. Ausgehend von Fallberichten zur Wirkung von frischen, grünen Hülsen bzw. getrockneten Samen, die traditionell in Südeuropa in gekochtem $\mathrm{Zu}$ stand verzehrt werden, wurden in offenen, vergleichenden Studien die Wirkung von V.faba-Extrakten und «synthetischem» L-Dopa in Kombination mit einem peripheren Decarboxylase-Hemmer bei Einmalgabe untersucht. Sowohl nach Einnahme von «synthetischem» L-Dopa als auch eines V.-faba-Extraktes wurden signifikante Verbesserungen der motorischen Symptome infolge eines M. Parkinsons bei guter Verträglichkeit berichtet. Die Anwendung von $V$. faba wird durch Begleitstoffe begrenzt, die für den Favismus (genetisch bedingte Form einer hämolytischen Anämie), Lathyrismus (Neurotoxizität durch Nitril-haltige Aminosäuren) oder akute Anämien verantwortlich sind. In einer weiteren Studie am Menschen wurde die Wirksamkeit von V.-fabaSprossen bei Parkinson-Patienten im Vergleich zu «synthetischem» L-Dopa in Kombination mit einem peripheren Decarboxylase-Hemmer nach einmaliger Gabe untersucht.
Die 13 Tage alten Sprossen (Tag 9 nach der Keimung) enthielten ca. 3-5\% L-Dopa (bezogen auf das Trockengewicht). In der V.-fabaGruppe wurden, wie in der L-DopaGruppe, signifikante Verbesserungen motorischer Symptome bei guter Verträglichkeit beobachtet. Mögliche Begleitstoffe, die zu unerwünschten Wirkungen führen könnten, sind in den Sprossen in weit geringeren Mengen enthalten als in den Samen oder den Hülsen.

Mucuna pruriens var. pruriens (Fabaceae), die Juckbohne, stammt aus Indien und ist seit Jahrtausenden in der indischen Ayurveda bekannt. Die Samendroge enthält L-Dopa in Mengen von 5-6\%, daneben Tetrahydroisochinoline und TryptaminDerivate. Tierexperimentelle Studien belegen eine hohe Wirksamkeit von M.-pruriens-Extrakten in verschiedenen, etablierten Tiermodellen. In einer offenen Beobachtungsstudie wurde nach durchschnittlich $20 \mathrm{Mo-}$ naten Behandlung mit einem M.-pruriens-Extrakt eine gute Wirksamkeit bei einer guten Verträglichkeit gefunden. Eine gute Wirksamkeit wurde auch in weiteren Studien beobachtet. Der Referent kam zum Schluss, dass Extrakte aus V. faba und besonders aus M. pruriens (aufgrund des höheren L-Dopa-Gehaltes und möglicher neuroprotektiver Eigenschaften) in der Zukunft eine wichtige Rolle in der symptomatischen The-

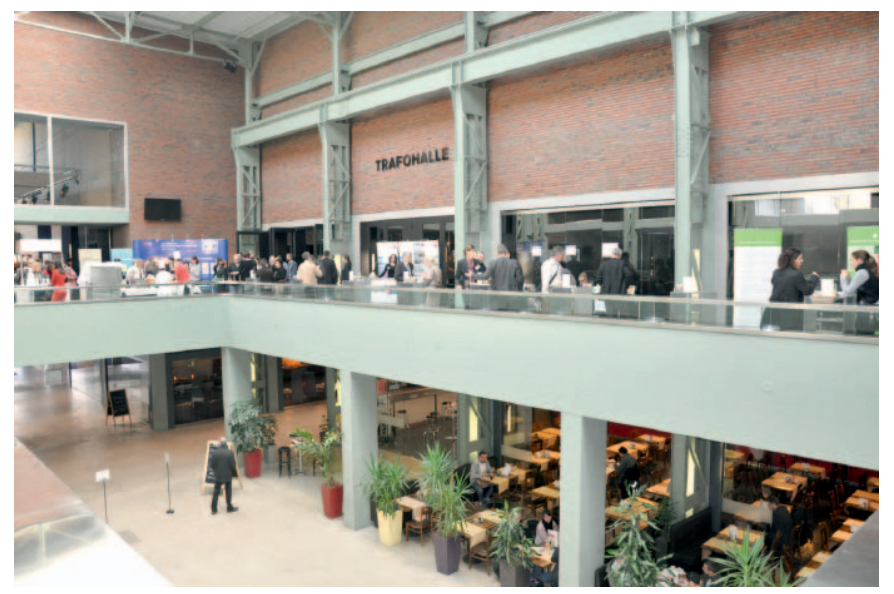

Abb. 3. Die Teilnehmenden fühlten sich in den grosszügigen Hallen des Trafo in Baden wohl.

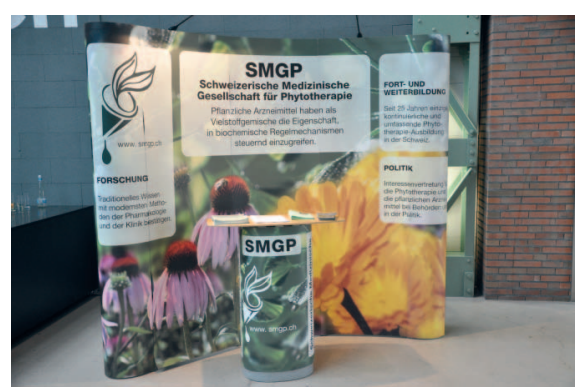

Abb. 4. Die SMGP organisiert als wissenschaftliche Fachgesellschaft jährlich die Schweizerische Tagung für Phytotherapie mit grossem Erfolg.

rapie des M. Parkinson spielen könnten und eventuell eine besser verträgliche Alternative zu «synthetischem» L-Dopa darstellen. Die Forschung stünde vor grossen Herausforderungen, sind doch derzeit nur Zubereitungen aus dem Internet vorwiegend aus den USA erhältlich. Damit verbunden sind die Unsicherheiten bezüglich der Qualität. Leider ist nicht davon auszugehen, dass die Forschung in den nächsten Jahren grosse Fortschritte machen wird. Die Fördermittel dazu fehlen.

\section{Bilanz der Tagung}

Dr. med. Robert Käufeler stellte in seiner Bilanz der Tagung fest, dass eine Fülle von Informationen zusammengekommen ist. Die Referierenden konnten die Neugier für das Thema durchwegs wecken. Er forderte eine vermehrte Fokussierung der Forschung auf die angesprochenen Themen und äusserte die Hoffnung, es würden vermehrt Firmen den Mut finden, neue Gebiete für die Phytotherapie zu erschliessen und mit entsprechenden Produkten aufzuwarten.

Die Tagung wurde wie in früheren Jahren im Kongresszentrum Trafo (Abb. 3) in Baden durchgeführt. Eine perfekte Infrastruktur mit delikater Verpflegung beim Steh-Lunch sorgte auch diesmal für eine besondere Stimmung. Die Möglichkeiten zum 


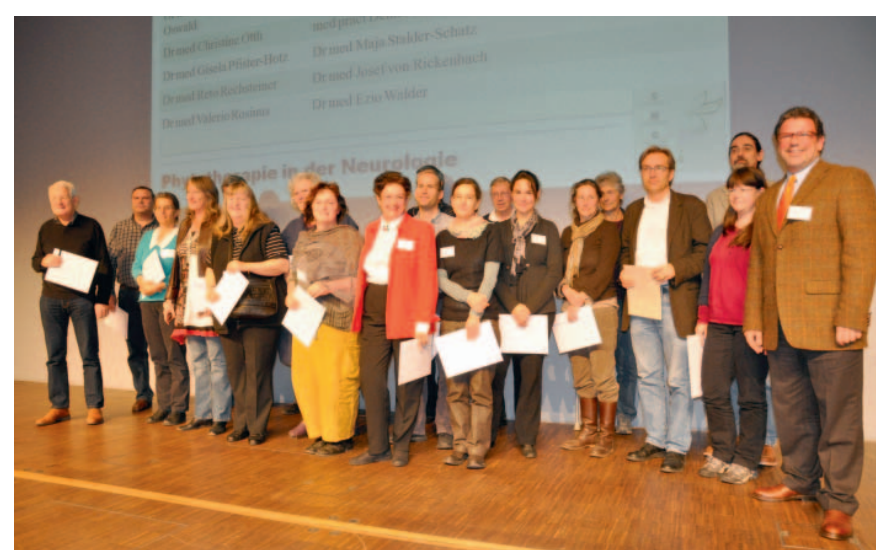

Abb. 5. Nach der Anerkennung des Fähigkeitsprogramms Phytotherapie (SMGP) durch das Schweizerische Institut für ärztliche Fortund Weiterbildung (SIFW) haben im Rahmen der Übergangsordnung 53 Ärztinnen und Ärzte die Bestätigung als dessen Absolventen erhalten. Sie unterliegen weiterhin einer Weiterbildungspflicht. Viele von ihnen waren an der Tagung dabei und übernahmen den Fähigkeitsausweis persönlich.

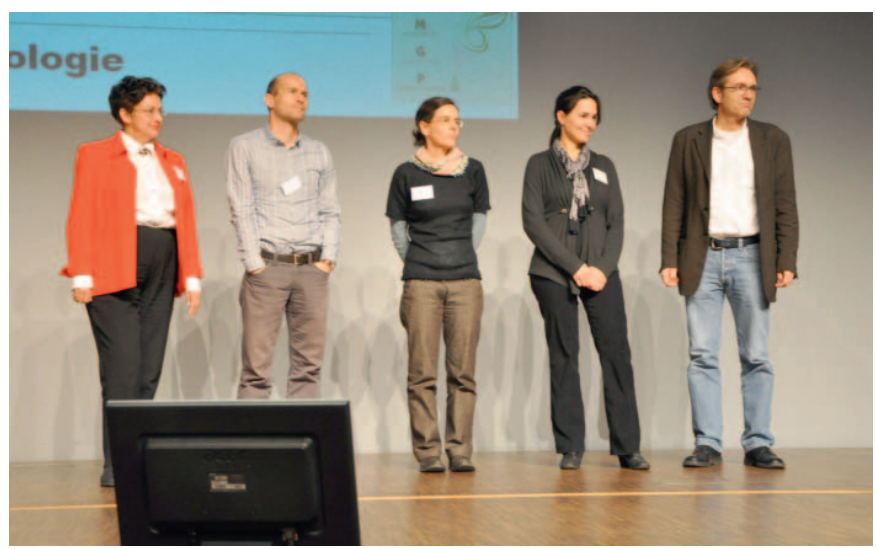

Abb. 6. Fünf Mitglieder der SMGP wurden erstmals mit dem Fähigkeitsausweis bzw. dem Zertifikat Phytotherapie nach absolviertem Fähigkeitsprogramm ausgezeichnet. vielseitigen Gedankenaustausch waren vom Programm her gegeben und wurden ausgiebig genutzt. Die Arzneipflanzen verarbeitende Industrie präsentierte ihre Produkte im Rahmen der Industrieausstellung, sodass ein guter Überblick über die Phytotherapie in der Schweiz gewonnen werden konnte. Die nächste Schweizerische Jahrestagung für Phytotherapie der Schweizerischen Medizinischen Gesellschaft für Phytotherapie (SMGP) (Kasten, Abb. 4-6) findet am 22. November 2012 statt. Das Thema ist attraktiv: «Infektionskrankheiten - Leadership für Phytotherapie?».

\section{Dank}

Der Bericht wurde mithilfe der ausführlichen Kurzfassungen der Referierenden zusammengestellt. Der Tagungsband kann bei der Korrespondenzadresse gegen ein kleines Entgelt bestellt werden. Die vollständigen Texte und die Portraits der Referierenden sind publiziert auf www.smgp.ch/archiv/archivxfiles/jtg.html. 